\title{
Caracterización morfoagronómica de la colección de germoplasma de ají dulce (Capsicum spp.) del Caribe colombiano
}

\section{Morphoagronomic characterization of $\mathbf{t}$ sweet pepper (Capsicum spp.) germplasm collection in the colombian Caribbean}

\author{
Ender M. Correa-Álvarez ${ }^{* \bullet}$; Rommel I. León-Pacheco'®®; Miguel A. Lobato-Ureche ${ }^{1 \oplus ;}$ \\ Mario A. García-Davila² ${ }^{\circledR}$; Carlos G. Muñoz-Perea ${ }^{\circledR}$; Hermes Aramendiz-Tatis ${ }^{3}{ }^{\circledR}$ \\ Recibido para publicación: Marzo 4 de 2019 - Aceptado para publicación: Mayo 24 de 2019
}

\begin{abstract}
RESUMEN
El ají dulce tipo topito es considerado una las principales hortalizas en la región Caribe de Colombia debido a su tradición productiva y consumo cotidiano en al menos seis de los siete departamentos de la costa atlántica. En este sentido el desarrollo de líneas investigación en mejoramiento genético que conduzcan a la obtención de cultivares de mayor productividad cobran gran relevancia. Por ello, con el fin de determinar la variabilidad morfológica y agronómica de la colección de germoplasma de ají dulce del Caribe colombiano y utilizarla en programas de mejoramiento genético, se evaluaron 45 descriptores (cualitativos y cuantitativos) de Capsicum spp. en 125 accesiones procedentes de los siete departamentos de la región Caribe de colombia. Para ello, se empleó un diseño completamente al azar en nueve plantas. El análisis de los datos se realizó a través del método de clasificación de Ward utilizando la distancia de Gower. Los resultados revelan polimorfismo en 19 características cualitativas, con una variabilidad del $67,9 \%$. El análisis mixto de los datos permitió separar las especies del género Capsicum de la colección en estudio; así mismo, la variabilidad fenotípica de la colección muestra potencial para su uso en programas de fitomejoramiento genético en caracteres de interés como calidad de fruto, arquitectura de planta y producción.
\end{abstract}

Palabras clave: Datos mixtos; Descriptores; Índice de gower; Recursos fitogenéticos; Variabilidad fenotípica.

${ }^{1}$ Corporación Colombiana de Investigación AgropecuariaAgrosavia, Centro de Investigación Caribia, Zona Bananera (Colombia).

${ }^{2}$ Universidad Nacional de Colombia Sede Palmira; Facultad de Ciencias Agropecuarias, Palmira (Colombia).

${ }^{3}$ Universidad de Córdoba, Facultad de Ciencias Agrícolas, Montería (Colombia).

*Autor para correspondencia: Ender Correa

Email: ecorrea@agrosavia.co

\begin{abstract}
"Topito" sweet pepper is considered one of the main vegetables in the colombian Caribbean region due to tradition and daily consumption in at least six of the seven continental departments of the Atlantic coast. Development of research lines for genetic improvement to obtain increased production cultivars are highly relevant. To determine the morphological and agronomic variability of the sweet pepper germplasm collection in the colombian Caribbean to use it for genetic improvement, a characterization with 45 qualitative and quantitative descriptors for Capsicum spp. in 125 accessions for the colombian Caribbean was carried out. A complete randomized design was used for nine plants. Data analysis was done through Ward's classification method using Gower's distance. Results showed polymorphism in 19 qualitative characteristics, with $67.9 \%$ variability. Data mixed analysis allowed to separate Capsicum genus species of the collection; likewise, collection phenotypic variability has potential for using in genetic breeding programs for desirable characters such as fruit quality, plant architecture and production.
\end{abstract}

Key words: Mixed data; Descriptors; Gower index; Plant genetic resources; Phenotypic variability.

\section{Cómo cita}

Correa-Álvarez, E., León-Pacheco, R., Lobato-Ureche, M. García-Davila, M., Muñoz-Perez, C. y Aramendiz-Tatis, H. 2019. Caracterización morfoagronómica de la colección de germoplasma de ají dulce (Capsicum spp.) del Caribe colombiano. Temas Agrarios, 24(2):81-95. https://doi.org/10.21897/rta.v24i2.1998

Temas Agrarios 2019. Este artículo se distribuye bajo lo términos de la Licencia Creative Commons Attrubution 4.0 (https://creativecommons.org/licenses/by-nc/4.0/deed.es), que permite copiar, redistribuir, remezclar, transformar y crear a partir del material, de forma no comercial, dando crédito y licencia de forma adecuada a los autores de la obra. 


\section{INTRODUCCIÓN}

Los ajíes son unas de las hortalizas de mayor consumo a nivel mundial; su comercialización ha registrado un crecimiento positivo debido a su diversificación en usos alimenticios, industriales, medicinales y ornamentales (Duran, 2013; Srivastava y Mangal, 2019; Costa et al., 2019). En la región Caribe de Colombia se estiman unos 4.284 productores con unidades productivas en promedio de 0,25 ha en cultivos de ajíes dulce tipo topito, que se han consolidado como actividad económica rural debido a la gran aceptación del producto en el mercado regional por sus características organolépticas (sabor, aroma y color) y diversos usos en la preparación de platos típicos de la gastronomía costeña (MINCIT, 2013; Pinto et al., 2013; Agronet, 2019).

A nivel mundial para el año 2017, las estadísticas oficiales para ajíes (chiles y pimientos) indican una producción de 36 millones de toneladas producidas en 1,98 millones de hectáreas, con rendimientos promedios de $7 \mathrm{t} \mathrm{ha}^{-1}$; en donde Colombia se posiciona en los puestos 89, 52 y 66 en producción, área cosechada y rendimientos respectivamente (FAOSTAT, 2019). En Colombia, las estadísticas para el año 2018 reportadas por Agronet (Agronet, 2019) en ajíes dulces señalan una producción de 7.363 toneladas producidas en 1.071 hectáreas en la región Caribe y con rendimientos medios de

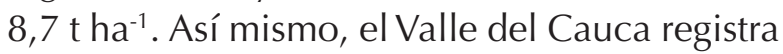
rendimientos medios de 25,3 $\mathrm{tha}^{-1}$ producto del uso de variedades mejoradas e implementación de paquetes tecnológicos, lo que evidencia los rezagos del cultivo en la región Caribe y a que a su vez generan demandas y oportunidades de investigación en fitomejoramiento y manejo agronómico para el cultivo de ají dulce.

El valor de los recursos fitogenéticos radica en su utilización para la generación de nuevos cultivares con características agronómicas superiores y de interés. En este sentido, los estudios de caracterización cumplen el propósito de dar a conocer las variantes génicas presentes en las colecciones de germoplasma para posteriormente ser empleadas en programas de mejoramiento genético vegetal (Vallejo y Estrada, 2013; Hidalgo y Vallejo, 2014).

América es considerado como el centro de origen de las especies cultivadas del género Capsicum, el cual comprende los pimentones y ajíes (picantes y dulces); este género tiene más 73 mil accesiones conservadas a nivel mundial, y donde Colombia posee alrededor del 1\% del germoplasma (FAO, 2010; Barchenger y Bosland, 2019; Barchenger et al., 2019; Srivastava y Mangal, 2019). Así mismo, el germoplasma de Capsicum ha sido objeto de diversos estudios de caracterización morfológica, agronómica, bioquímica, molecular y funcional entre otros, tanto a nivel mundial como en Colombia, a fin de identificar accesiones que sirvan como fuentes de genes en el mejoramiento de características para la resistencia a estreses abióticos y bióticos y de otros rasgos de interés (contenidos de capsaicina, carotenoides, formas, tamaños, colores, etc.) para las diferentes agroindustrias demandantes de ajíes (Villota-Cerón et al., 2012, Araújo et al., 2019; Guzmán et al., 2019; Jesus et al., 2019; Mendes et al., 2019; Rahevar et al., 2019).

La caracterización de recursos fitogenéticos requiere del manejo y análisis de un gran número de mediciones sobre cada accesión del germoplasma; razón por la cual, es usual la utilización de métodos multivariados, ya que estos permiten el análisis de las accesiones incluyendo múltiples características de manera simultánea y sin dejar de considerar la relación existente entre ellas. Generalmente, la caracterización de germoplasma involucra el registro de variables de naturaleza cuantitativa y cualitativa. El índice de Gower, admite realizar un análisis unificado de descriptores, es decir, descriptores de tipo cuantitativo y cualitativo (mixtos) simultáneamente. Así mismo, dentro 
de la gama de análisis multivariados, el método Ward, permite la generación de grupos lo más homogéneos posible, para clasificar las accesiones en estudio (Franco e Hidalgo, 2003).

Considerando la importancia de los ajíes dulces en la región y la necesidad de documentar y generar valor agregado al recurso genético colectado; esta investigación tuvo como objetivo caracterizar morfo-agronómicamente el germoplasma de ajíes dulces colectados en la región Caribe colombiana con fines de uso para desarrollar un programa de mejoramiento genético de la especie que conduzca al desarrollo de nuevos cultivares con características agronómicas superiores al germoplasma utilizado actualmente por los productores de la región.

\section{MATERIALES Y MÉTODOS}

La investigación se desarrolló en Agrosavia Cl. Caribia, ubicado en Zona Bananera (Magdalena), con coordenadas $10^{\circ} 47^{\prime} \mathrm{N}$ y $74^{\circ} 10^{\prime} \mathrm{W}$, altitud de 18 m.s.n.m., temperatura media de $28^{\circ} \mathrm{C}$, humedad relativa del $82 \%$, precipitación media anual de $1280 \mathrm{~mm}$ y zona de vida de bosque seco tropical. El recurso genético correspondió a 125 accesiones de ají dulce que conforman la colección de germoplasma de Agrosavia $\mathrm{Cl}$. Caribia colectada en 2013 en siete departamentos de la región Caribe colombiana (Tabla 1).

El experimento se estableció bajo un diseño completamente al azar con nueve observaciones por accesión. La unidad experimental, constó de una planta sembrada a $1 \times 1 \mathrm{~m}$ entre plantas y surcos respectivamente; así mismo, para asegurar la uniformidad de la información, se sembraron surcos bordes y los datos experimentales se tomaron en plantas en plena competencia. Se utilizaron los descriptores para Capsicum spp. (IPGRI et al., 1995), registrando 45 caracteres (28 cualitativos y 17 cuantitativos) responsables de la morfología, arquitectura de la planta y características relacionadas con aspectos agronómicos. Para el análisis de los datos, se descartaron los descriptores que no presentaron variación; para las variables cuantitativas y cualitativas se utilizaron los promedios y modas respectivamente. El análisis multivariado de los datos se realizó mediante el método de clasificación de Ward utilizando la distancia de Gower, con el uso del programa estadístico infoStat, versión 2017 (Di Rienzo et al., 2017).

\section{RESULTADOS Y DISCUSIÓN}

Nueve descriptores fueron descartados del análisis multivariado por no presentar variación: forma tallo (cilíndrica), color del tallo (verde), forma de la corola (rotácea), pigmentación del cáliz (ausente), manchas antocianínicas (ausente), cuello en la base del fruto (ausente), apéndice del fruto vestigio de la floración (ausente), color del fruto en estado intermedio (verde) y color de la semilla (amarillo) (Tabla 2). Al respecto, Votava et al. (2005) y Paran y Van Der Knaap (2007) señalan que en muchas regiones es común por parte de los agricultores, realizar ciclos de selección hacia atributos morfológicos y agronómicos de interés (pungencia, forma, tamaño, peso y color de fruto) en los cultivares locales de Capsicum.

En este sentido, estudios realizados por Medina et al. (2006) y Nicolaï et al. (2013) indican que el principal efecto negativo de las selecciones artificiales en Capsicum es la reducción en su base genética; por tanto, la ausencia de variabilidad en atributos cualitativos puede ser explicada por procesos de selección direccional hacia atributos deseables por los productores, lo que generaría una reducción en la diversidad de las características fenotípicas como consecuencia de procesos de domesticación, efecto fundador y deriva genética; complementariamente Marame et al. (2009), Tang et al. (2010) y Occhiuto et al. (2014), señalan que el nivel de variación dentro 
de las especies domesticadas de Capsicum es menor que en sus parientes silvestres. Lo descrito anteriormente, podría ser el caso de los ajíes dulces de la región Caribe de Colombia; donde los principales cultivares corresponden a los denominados regionalmente como "ají topito" y "ají chino", que son los tradicionalmente cultivados y seleccionados por los agricultores; por ende, en este escenario muchas características morfológicas de interés podrían haber quedado fijadas en estos materiales como resultado de procesos continuos de selección. Se encontró que 19 de los 28 descriptores cualitativos presentaron polimorfismo, lo que representa un $67,9 \%$ de atributos con variabilidad. Al considerar el número de variantes o estados totales de los descriptores evaluados, es decir, 126 morfolalelos, se detectaron 71 en las accesiones del estudio, lo que representa un 56,4\% de los polimorfismos cualitativos (Tabla 2). El moderado polimorfismo encontrado, podría explicarse debido al hecho de que 123 de las accesiones de la colección $(98,4 \%)$ corresponden a la especie Capsicum chinense (cultivar ají topito) y solamente dos accesiones (AjCo62 y AjMa03) pertenecen a la especie Capsicum annuum (cultivar ají chino) (Tabla 1).

Tabla 1. Colección de germoplasma de Capsicum (125 accesiones), Agrosavia Cl Caribia.

\begin{tabular}{|c|c|c|c|c|c|c|c|c|c|}
\hline Municipio, Departamento & Especie & \multicolumn{4}{|c|}{ Código Accesión } & $\begin{array}{c}\text { Altitud } \\
\text { (m.s.n.m) }\end{array}$ & $\begin{array}{l}\text { Temp } \\
\left({ }^{\circ} \mathrm{C}\right)\end{array}$ & $\begin{array}{l}\text { Pma } \\
(\mathrm{mm})\end{array}$ & C.C. \\
\hline Luruaco, Atlántico & C. chinense & AjAt01 & & & & 40 & 27,8 & 1148 & Aw \\
\hline Manatí, Atlántico & C. chinense & AjAt03 & AjAt02 & & & 7 & 28,5 & 1004 & Aw \\
\hline Suán, Atlántico & C. chinense & AjAt05 & AjAt07 & AjAt04 & AjAt06 & 7 & 28,5 & 1079 & Aw \\
\hline Hatillo de Loba, Bolívar & C. chinense & AjBo01 & & & & 28 & 28,3 & 2261 & $\mathrm{Am}$ \\
\hline Carmen de Bolívar, Bolívar & C. chinense & AjBo02 & & & & 158 & 26,9 & 1179 & Aw \\
\hline Codazzi, Cesar & C. chinense & AjCe03 & $\mathrm{AjCe} 04$ & & & 135 & 28,1 & 1560 & Aw \\
\hline El Copey, Cesar & C. chinense & AjCe01 & AjCe02 & & & 136 & 27,5 & 1369 & Aw \\
\hline \multirow[t]{12}{*}{ Cereté, Córdoba } & C. chinense & AjCo01 & $\mathrm{AjCo} 27$ & $\mathrm{AjCo} 40$ & AjCo52 & 15 & 27,7 & 1264 & Aw \\
\hline & & AjCo05 & AjCo28 & AjCo41 & AjCo53 & & & & \\
\hline & & AjCo13 & AjCo29 & $\mathrm{AjCo} 42$ & AjCo54 & & & & \\
\hline & & AjCo14 & AjCo30 & $\mathrm{AjCo} 43$ & AjCo55 & & & & \\
\hline & & AjCo15 & AjCo31 & $\mathrm{AjCo} 44$ & AjCo56 & & & & \\
\hline & & AjCo19 & AjCo32 & AjCo45 & $\mathrm{AjCo} 57$ & & & & \\
\hline & & AjCo20 & $\mathrm{AjCo} 34$ & AjCo46 & AjCo58 & & & & \\
\hline & & AjCo21 & AjCo35 & $\mathrm{AjCo} 47$ & AjCo60 & & & & \\
\hline & & $\mathrm{AjCo} 22$ & AjCo36 & $\mathrm{AjCo} 48$ & AjCo85 & & & & \\
\hline & & AjCo23 & AjCo37 & AjCo49 & AjCo86 & & & & \\
\hline & & $\mathrm{AjCo} 24$ & AjCo38 & $\mathrm{AjCo} 50$ & & & & & \\
\hline & & AjCo26 & AjCo39 & AjCo51 & & & & & \\
\hline \multirow[t]{2}{*}{ Canalete, Córdoba } & C. chinense & AjCo79 & AjCo80 & AjCo81 & AjCo82 & 52 & 26,8 & 1338 & Aw \\
\hline & & AjCo83 & AjCo84 & & & & & & \\
\hline \multirow[t]{2}{*}{ Lorica, Córdoba } & C. chinense & AjCo67 & AjCo68 & AjCo69 & $\mathrm{AjCo} 70$ & 14 & 27,6 & 1270 & Aw \\
\hline & & AjCo71 & AjCo72 & $\mathrm{AjCo} 74$ & & & & & \\
\hline Los Córdobas, Córdoba & C. chinense & AjCo78 & & & & 8 & 26,9 & 1509 & Aw \\
\hline \multirow[t]{2}{*}{ Montería, Córdoba } & C. chinense & AjCo02 & $\mathrm{AjCo07}$ & AjCo10 & AjCo25 & 20 & 27,4 & 1225 & Aw \\
\hline & & AjCo03 & AjCo08 & AjCo11 & AjCo59 & & & & \\
\hline
\end{tabular}


Correa-Álvarez et al. - Caracterización morfoagronómica de ají dulce.

Continuación Tabla 1. Colección de germoplasma de Capsicum (125 accesiones), Agrosavia Cl Caribia.

\begin{tabular}{|c|c|c|c|c|c|c|c|c|c|}
\hline \multirow[t]{2}{*}{ Municipio, Departamento } & \multirow[t]{2}{*}{ Especie } & \multicolumn{4}{|c|}{ Código Accesión } & \multirow{2}{*}{$\begin{array}{c}\text { Altitud } \\
\text { (m.s.n.m) }\end{array}$} & \multirow[t]{2}{*}{$\begin{array}{c}\text { Temp } \\
\left({ }^{\circ} \mathrm{C}\right)\end{array}$} & \multirow{2}{*}{$\begin{array}{l}\text { Pma } \\
(\mathrm{mm})\end{array}$} & \multirow[t]{2}{*}{ C.C. } \\
\hline & & AjCo06 & AjCo09 & AjCo12 & AjCo61 & & & & \\
\hline & & AjCo63 & & & & & & & \\
\hline & C. annuum & AjCo62 & & & & & & & \\
\hline $\begin{array}{c}\text { San Bernardo del Viento, } \\
\text { Córdoba }\end{array}$ & C. chinense & AjCo75 & AjCo77 & & & 8 & 27,5 & 1365 & Aw \\
\hline San Carlos, Córdoba & C. chinense & AjCo65 & AjCo64 & AjCo66 & & 15 & 27,7 & 1460 & Aw \\
\hline San Pelayo, Córdoba & C. chinense & AjCo16 & $\mathrm{AjCo} 17$ & & & 10 & 27,6 & 1349 & Aw \\
\hline Barrancas, La Guajira & C. chinense & AjGu01 & AjGu02 & & & 158 & 27,5 & 1172 & Aw \\
\hline \multirow[t]{2}{*}{ Dibulla, La Guajira } & C. chinense & AjGu06 & AjGu07 & AjGu08 & AjGu09 & 10 & 28,3 & 1426 & Aw \\
\hline & & AjGu10 & AjGu11 & & & & & & \\
\hline Ciénaga, Magdalena & C. chinense & AjMa07 & AjMa08 & AjMa10 & & 6 & 28 & 622 & BSh \\
\hline Fundación, Magdalena & C. chinense & AjMa11 & & & & 45 & 27,9 & 1390 & Aw \\
\hline Remolino, Magdalena & C. chinense & AjMa05 & AjMa06 & & & 75 & 28,2 & 964 & Aw \\
\hline \multirow[t]{2}{*}{ Sitio Nuevo, Magdalena } & C. annuum & АjMa03 & & & & 6 & 28,2 & 906 & Aw \\
\hline & C. chinense & AjMa04 & & & & & & & \\
\hline $\begin{array}{l}\text { Zona Bananera, Magda- } \\
\text { lena }\end{array}$ & C. chinense & AjMa02 & & & & 26 & 27,8 & 1356 & Aw \\
\hline Buenavista, Sucre & C. chinense & AjSu01 & & & & 95 & 27,4 & 1152 & Aw \\
\hline \multirow[t]{3}{*}{ Ovejas, Sucre } & C. chinense & AjSu02 & AjSu08 & AjSu11 & AjSu14 & 255 & 26,3 & 1294 & Aw \\
\hline & & AJSu03 & AjSu09 & AjSu12 & & & & & \\
\hline & & AjSu06 & AjSu10 & AjSu13 & & & & & \\
\hline San Marcos, Sucre & C. chinense & AjSu04 & & & & 25 & 27,8 & 1858 & Aw \\
\hline Sincelejo, Sucre & C. chinense & AjSu05 & & & & 202 & 26,6 & 1164 & Aw \\
\hline Sucre, Sucre & C. chinense & AjSu07 & & & & 17 & 27,9 & 2472 & $\mathrm{Am}$ \\
\hline
\end{tabular}

Temp: temperatura; Pma: precipitación media anual; C.C: clasificación climática de Köppen - Geiger. Aw: clima de sabana tropical; Am: clima tropical del monzón; BSh: clima semiárido caliente.

En este sentido, diversos investigadores como Esbaugh (1993) y Pickersgill (1997); indican que C. annuum y C. chinense corresponden a las especies de mayor dispersión mundial del género, siendo C. annuum la más ampliamente sembrada y domesticada; sin embargo, por su baja adaptación a trópicos húmedos bajos, es reemplazada en estas áreas por las especies C. chinense y C. frutenscens. Así mismo, autores como Votava et al. (2002) señalan que uno de los objetivos de la conservación ex situ, es mantener tantos alelos como sea factible, a fin de mantener la mayor variabilidad genética en conjuntos de accesiones del menor tamaño posible. La tabla 3, muestra el agrupamiento de las accesiones utilizando variables cuantitativas y cualitativas, es decir, datos mixtos (continuos y categóricos). Se determinaron cinco grupos con 40, 37, 20, 26 y 2 accesiones para los grupos GI, GII, GIII, GIV y GV respectivamente. El GI incluye accesiones provenientes de cinco de los siete departamentos donde se efectuó la colecta. Estos departamentos son: Córdoba, Atlántico, Bolívar, Cesar y Sucre con 35, 2, 1, 1 y 1 accesiones respectivamente. Así mismo, los atributos cualitativos por lo que se caracteriza principalmente este grupo son: habito de crecimiento $(\mathrm{HC})$ erecto, antocianina del nudo (AN) verde, margen de la lámina foliar (MLF) ciliada, color de la hoja $(\mathrm{CH})$ verde oscuro, 
pubescencia en las hojas $(\mathrm{PH})$ densa, forma de la hoja (FH) oval, posición de las flores (PF) erecta, color de la corola (CC) verde claro, margen del cáliz $(M C)$ dentado, constricción anular del cáliz (CAC) presente, color de las anteras (CA) verde oscuro, exserción del estigma (EE) al mismo nivel, color del filamento (CF) morado, forma del fruto (FF) intermedio, forma del ápice del fruto (FAF) puntudo, forma del fruto con la unión con el pedicelo (FFUP) truncado, epidermis del fruto (EF) lisa, color del fruto en estado maduro (CFM) rojo y arrugamiento transversal del fruto (ATF) intermedio. Estados únicos ó morfolalelos presentes en un único individuo de la colección de estudio, fueron encontrados en este grupo para los descriptores: CA verde claro (AjCo66), CF lila (AjCo01) y azul (AjCo56) y EF semirrugosa (AjCo02) (Tabla 4).

Tabla 2. Descriptores cualitativos registrados en 125 accesiones de Capsicum spp de la colección de germoplasma de Agrosavia Cl Caribia.

\begin{tabular}{lccc}
\hline Descriptor & $\begin{array}{c}\text { Moda } \\
\text { general }\end{array}$ & $\begin{array}{c}\text { Estados } \\
\text { Descriptor }\end{array}$ & $\begin{array}{c}\text { Estados } \\
\text { Encontrados }\end{array}$ \\
\hline Habito de crecimiento HC & Compacta & 4 & 3 \\
Forma del tallo FT & Cilíndrico & 3 & $\mathbf{1}$ \\
Color del tallo CT & Verde & 4 & $\mathbf{1}$ \\
Antocianina del nudo AN & Verde & 4 & 3 \\
Margen de la lámina foliar MLF & Ciliada & 3 & 2 \\
Color de la hoja CH & Verde claro & 8 & 3 \\
Pubescencia en las hojas PH & Densa & 3 & 3 \\
Forma de la hoja FH & Oval & 3 & 3 \\
Forma de la corola FC & Rotácea & 3 & $\mathbf{1}$ \\
Pigmentación en el cáliz PC & Ausente & 2 & $\mathbf{1}$ \\
Color de la corola CC & Verde pálido & 9 & 2 \\
Margen del cáliz MC & Dentado & 4 & 2 \\
Constricción anular del cáliz CAC & Presente & 2 & 2 \\
Color de las anteras CA & Verde oscuro & 9 & 6 \\
Exserción del estigma EE & Al mismo nivel & 3 & $\mathbf{3}$ \\
Color del filamento CF & Morado & 7 & $\mathbf{1}$ \\
Manchas anticanónicas MA & Ausente & 2 & $\mathbf{1}$ \\
Posición de las flores PF & Erecta & 3 & $\mathbf{1}$ \\
Forma del fruto FF & Triangular & 6 & 3 \\
Cuello en la base fruto CBF & Ausente & 2 & 5 \\
Apéndice del fruto vestigio de la floración AFVF & Ausente & 2 & 3 \\
Forma del ápice del fruto FAF & Puntudo & 5 & 3 \\
Epidermis del fruto EF & Lisa & 3 & 3 \\
\hline Valoresen & & 3 \\
\hline
\end{tabular}

*Valores en negrilla corresponden a descriptores que no presentaron polimorfismo en la colección de germoplasma de Capsicum. 
Continuación Tabla 2. Descriptores cualitativos registrados en 125 accesiones de Capsicum spp de la colección de germoplasma de Agrosavia Cl Caribia.

\begin{tabular}{lccc}
\hline Descriptor & $\begin{array}{c}\text { Moda } \\
\text { general }\end{array}$ & $\begin{array}{c}\text { Estados } \\
\text { Descriptor }\end{array}$ & $\begin{array}{c}\text { Estados } \\
\text { Encontrados }\end{array}$ \\
\hline Forma del fruto en la unión con el pedicelo FFUP & Truncado & 5 & 4 \\
Color del fruto maduro CFM & Rojo & 13 & 4 \\
Color fruto estado intermedio CFI & Verde & 7 & $\mathbf{1}$ \\
Arrugamiento transversal del fruto ATF & Intermedio & 3 & 3 \\
Color de la semilla CS & Amarillo & 4 & $\mathbf{1}$ \\
TOTAL & & $\mathbf{1 2 6}^{*}$ & $\mathbf{7 1 *}^{*}$ \\
\hline
\end{tabular}

*Valores en negrilla corresponden a descriptores que no presentaron polimorfismo en la colección de germoplasma de Capsicum.

El Gll comprende accesiones provenientes de seis departamentos, los cuales son: Córdoba, Guajira, Cesar, Atlántico, Sucre y Magdalena con 21, 5, 4, 3, 3 y 1 accesiones respectivamente. Los atributos cualitativos por lo que se caracteriza este grupo principalmente son: $\mathrm{HC}$ compacto, AN verde, MLF ciliada, $\mathrm{CH}$ verde claro, $\mathrm{PH}$ densa, $\mathrm{FH}$ oval, PF erecta, $\mathrm{CC}$ verde claro, $\mathrm{MC}$ dentado, CAC presente, CA morado claro, EE al mismo nivel, CF morado, FF intermedio, FAF puntudo, FFUP truncado, EF lisa, CFM rojo y ATF intermedio (Tabla 4).

El GIII abarca accesiones provenientes de los departamentos de Córdoba, Atlántico, Guajira y Sucre con 16, 2, 1 y 1 accesiones respectivamente. Los caracteres cualitativos por lo que se destaca este grupo principalmente son: $\mathrm{HC}$ compacto, AN verde, MLF ciliada, $\mathrm{CH}$ verde claro, $\mathrm{PH}$ densa, $\mathrm{FH}$ oval, $\mathrm{PF}$ erecta, $\mathrm{CC}$ verde claro, $\mathrm{MC}$ dentado, CAC presente, CA verde oscuro, EE al mismo nivel y exserto, CF moradooscuro, FFtriangular, FAF puntudo, FFUP truncado, EF lisa, CFM rojo y ATF intermedio. Además, este grupo registra un estado único para el descriptor CFM café rojizo (AjCo09) (Tabla 4).

El GIV presenta accesiones provenientes de los departamentos de Sucre, Magdalena, Córdoba, Guajira y Bolívar con 9, 7, 7, 2 y 1 accesiones respectivamente. Los descriptores cualitativos por lo que se destaca este grupo principalmente son: HC compacto, AN verde, MLF entera, $\mathrm{CH}$ verde, $\mathrm{PH}$ intermedia, FH oval, PF erecta, CC verde claro, MC dentado, CAC presente, CA morado claro, EE exserto, CF morado, FF intermedio, FAF puntudo, FFUP truncado, EF lisa, CFM rojo y ATF intermedio (Tabla 4).

El GV incluye solamente dos accesiones provenientes de los departamentos de Córdoba y Magdalena. Los descriptores cualitativos por lo que se caracteriza este grupo principalmente son: HC compacto, AN verde y morado oscuro, MLF entera, $\mathrm{CH}$ verde claro, $\mathrm{PH}$ escasa, $\mathrm{FH}$ lanceolada, PF intermedia y pendiente, CC blanco, MC dentado, CAC ausente, CA verde oscuro, EE exserto, CF blanco, FF en bloque y acampanulado en bloque, FAF hundido $y$ hundido puntudo, FFUP cordado y lobulado, EF lisa, CFM rojo y ATF levemente corrugado. Así mismo, presenta estados únicos para los descriptores AN morado oscuro (AjCo62), PF pendiente (AjMa03) e intermedia (AjCo62), FF en bloque (AjCo62) y acampanulado en bloque (AjMa03), FAF hundido y puntudo (AjMa03) y FFUP cordado (AjMa03) y lobulado (AjCo62) (Tabla 4). 
Correa-Álvarez et al. - Caracterización morfoagronómica de ají dulce.

Tabla 3. Agrupamiento de 125 accesiones de la colección de germoplasma de Capsicum de Agrosavia Cl Caribia obtenidos a partir del método de Ward (1963) e índice de Gower (1971).

\begin{tabular}{|c|c|c|c|c|c|c|c|c|c|c|}
\hline Grupo & & & & & Acce & iones & & & & \\
\hline \multirow{4}{*}{ I } & AjAt01 & AjAt03 & AjBo02 & AjCe02 & AjCo01 & AjCo02 & AjCo03 & AjCo05 & AjCo06 & $\mathrm{AjCoO7}$ \\
\hline & AjCo17 & AjCo23 & AjCo25 & AjCo28 & AjCo31 & AjCo32 & AjCo35 & AjCo36 & AjCo37 & AjCo38 \\
\hline & AjCo41 & AjCo46 & $\mathrm{AjCo} 47$ & AjCo51 & AjCo53 & AjCo54 & AjCo56 & AjCo61 & AjCo63 & AjCo64 \\
\hline & AjCo66 & AjCo68 & AjCo69 & AjCo71 & AjCo72 & AjCo74 & AjCo75 & AjCo83 & AjCo85 & AjSu03 \\
\hline \multirow{4}{*}{ II } & AjAt02 & AjAt04 & AjAt06 & AjCe01 & AjCe03 & AjCe04 & AjCe04 & AjCo08 & AjCo11 & AjCo12 \\
\hline & AjCo13 & AjCo16 & AjCo20 & AjCo21 & AjCo24 & AjCo26 & AjCo30 & AjCo39 & AjCo43 & $\mathrm{AjCo} 44$ \\
\hline & AjCo45 & AjCo49 & AjCo52 & AjCo55 & AjCo58 & AjCo59 & AjCo78 & AjCo80 & AjGu01 & AjGu06 \\
\hline & AjGu07 & AjGu08 & AjGu09 & AjMa04 & AjSu09 & AjSu12 & AjSu14 & & & \\
\hline \multirow{2}{*}{ III } & AjAt05 & AjAt07 & AjCo09 & AjCo10 & AjCo19 & AjCo27 & AjCo29 & AjCo34 & AjCo40 & AjCo42 \\
\hline & AjCo57 & AjCo65 & AjCo67 & AjCo70 & AjCo77 & AjCo79 & AjCo82 & AjCo86 & AjGu10 & AjSu08 \\
\hline \multirow{3}{*}{ IV } & AjBo01 & AjCo14 & AjCo22 & AjCo48 & AjCo50 & AjCo60 & AjCo81 & AjCo84 & AjGu02 & AjGu11 \\
\hline & AjMa02 & AjMa05 & AjMa06 & AjMa07 & AjMa08 & AjMa10 & AjMa11 & AjSu01 & AjSu02 & AjSu04 \\
\hline & AjSu05 & AjSu06 & AjSu07 & AjSu10 & AjSu11 & AjSu13 & & & & \\
\hline V & $\mathrm{AjMa03}$ & AjCo62 & & & & & & & & \\
\hline
\end{tabular}

Tabla 4. Descriptores cualitativos de la colección de germoplasma de Capsicum spp. de Agrosavia Cl Caribia

\begin{tabular}{|c|c|c|c|c|c|c|}
\hline \multirow[b]{2}{*}{ Categoria } & \multicolumn{6}{|c|}{ Grupos } \\
\hline & $\begin{array}{c}\text { GI } \\
(40)\end{array}$ & $\begin{array}{l}\text { GII } \\
(37)\end{array}$ & $\begin{array}{l}\text { GIII } \\
(\mathbf{2 0 )}\end{array}$ & $\begin{array}{l}\text { GIV } \\
\text { (26) }\end{array}$ & $\begin{array}{l}\text { GV } \\
(2)\end{array}$ & $\begin{array}{c}\text { EU } \\
\text { (accesión) }\end{array}$ \\
\hline \multicolumn{7}{|l|}{ Habito de crecimiento } \\
\hline Compacta & 14 & 22 & 18 & 21 & 2 & \\
\hline Erecta & 24 & 12 & 2 & 5 & - & \\
\hline Intermedia & 2 & - & - & - & - & \\
\hline \multicolumn{7}{|l|}{ Antocianina del nudo } \\
\hline Verde & 38 & 29 & 18 & 23 & 1 & \\
\hline Morado claro & 2 & 8 & 2 & 3 & - & \\
\hline Morado oscuro & - & - & - & - & $1^{*}$ & AjCo62 \\
\hline
\end{tabular}

EU: estados únicos; GI, GII, GIII, GIV, GV: grupos obtenidos a partir del método de Ward (1963) e índice de Gower (1971); *valores resaltados corresponden a estados únicos encontrados en el germoplasma de estudio. 
Correa-Álvarez et al. - Caracterización morfoagronómica de ají dulce.

Continuación de Tabla 4. Descriptores cualitativos de la colección de germoplasma de Capsicum spp. de Agrosavia Cl Caribia

\begin{tabular}{|c|c|c|c|c|c|c|}
\hline \multirow{2}{*}{ Categoria } & \multicolumn{6}{|c|}{ Grupos } \\
\hline & $\begin{array}{c}\mathrm{GI} \\
(40)\end{array}$ & $\begin{array}{l}\text { GII } \\
(37)\end{array}$ & $\begin{array}{l}\text { GIII } \\
(20)\end{array}$ & $\begin{array}{l}\text { GIV } \\
\text { (26) }\end{array}$ & $\begin{array}{l}\text { GV } \\
(2)\end{array}$ & $\begin{array}{c}\text { EU } \\
\text { (accesión) }\end{array}$ \\
\hline \multicolumn{7}{|c|}{ Margen de la lámina foliar } \\
\hline Entera & 5 & 17 & 1 & 18 & 2 & \\
\hline Ciliada & 35 & 20 & 19 & 8 & - & \\
\hline \multicolumn{7}{|l|}{ Color de la hoja } \\
\hline Verde claro & 6 & 28 & 16 & 12 & 2 & \\
\hline Verde & 14 & 2 & 4 & 13 & - & \\
\hline Verde oscuro & 20 & 7 & - & 1 & - & \\
\hline
\end{tabular}

Pubescencia en las hojas

\begin{tabular}{lccccr} 
Escasa & 5 & 3 & 1 & 6 & 2 \\
Intermedia & 9 & 10 & 6 & 19 & - \\
Densa & 26 & 24 & 13 & 1 & - \\
\hline
\end{tabular}

Forma de la hoja

$\begin{array}{lllccc}\text { Deltoide } & 11 & 13 & 9 & 12 & - \\ \text { Oval } & 29 & 24 & 11 & 14\end{array}$

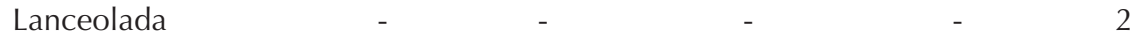

Posición de las flores

Pendiente

Intermedia

Erecta

$-$

$\begin{array}{llll}- & - & - & 1\end{array}$

1

AjMa03

40

37

20

$\begin{array}{ll}- & 1\end{array}$

AjCo62

Color de la corola

$\begin{array}{lccccc}\text { Blanco } & - & - & - & - & 2 \\ \text { Verde claro } & 40 & 37 & 20 & 26 & -\end{array}$

\section{Margen del cáliz}

$\begin{array}{lccccc}\text { Intermedio } & 2 & - & 7 & 3 & - \\ \text { Dentado } & 38 & 37 & 13 & 23 & 2\end{array}$

Constricción anular del cáliz

Ausente

$-$

40

37

20

2

EU: estados únicos; GI, GII, GIII, GIV, GV: grupos obtenidos a partir del método de Ward (1963) e índice de Gower (1971); *valores resaltados corresponden a estados únicos encontrados en el germoplasma de estudio. 
Correa-Álvarez et al. - Caracterización morfoagronómica de ají dulce.

Continuacion, Tabla 4. Descriptores cualitativos de la colección de germoplasma de Capsicum de Agrosavia Cl Caribia.

\begin{tabular}{|c|c|c|c|c|c|c|}
\hline \multirow[b]{2}{*}{ Categoria } & \multicolumn{6}{|c|}{ Grupos } \\
\hline & $\begin{array}{c}\text { GI } \\
(40)\end{array}$ & $\begin{array}{l}\text { GII } \\
(37) \\
\end{array}$ & $\begin{array}{l}\text { GIII } \\
(20)\end{array}$ & $\begin{array}{l}\text { GIV } \\
(26)\end{array}$ & $\begin{array}{l}\text { GV } \\
(2)\end{array}$ & $\begin{array}{c}\text { EU } \\
\text { (accesión) }\end{array}$ \\
\hline \multicolumn{7}{|l|}{ Color de las anteras } \\
\hline Morado claro & 10 & 28 & - & 12 & - & \\
\hline Negro & 1 & 1 & - & 2 & - & \\
\hline Verde oscuro & 27 & 7 & 19 & 11 & 2 & \\
\hline Verde intermedio & - & 1 & 1 & - & - & \\
\hline Verde claro & 1 & - & - & - & - & AjCo66 \\
\hline Amarillo claro & 1 & - & - & 1 & - & \\
\hline \multicolumn{7}{|l|}{ Exserción del estigma } \\
\hline Inserto & - & 2 & - & 1 & - & \\
\hline Al mismo nivel & 25 & 28 & 10 & 11 & - & \\
\hline Exserto & 15 & 7 & 10 & 14 & 2 & \\
\hline \multicolumn{7}{|l|}{ Color del filamento } \\
\hline Blanco & 1 & - & - & - & 2 & \\
\hline Morado & 35 & 36 & 5 & 21 & - & \\
\hline Lila & 1 & - & - & - & - & AjCo01 \\
\hline Morado oscuro & 2 & 1 & 15 & 5 & - & \\
\hline Azul & 1 & - & - & - & - & AjCo56 \\
\hline \multicolumn{7}{|l|}{ Forma del fruto } \\
\hline Alargado & 8 & 5 & 1 & 2 & - & \\
\hline Intermedio & 21 & 18 & 9 & 16 & - & \\
\hline Triangular (tipo topito) & 11 & 14 & 10 & 8 & - & \\
\hline Bloque & - & - & - & - & 1 & AjCo62 \\
\hline Acampanulado y en bloque & - & - & - & - & 1 & AjMa03 \\
\hline \multicolumn{7}{|l|}{ Forma del ápice del fruto } \\
\hline Puntudo & 23 & 31 & 15 & 21 & - & \\
\hline Romo & 14 & 3 & 5 & 4 & - & \\
\hline Hundido & 3 & 3 & - & 1 & 1 & \\
\hline Hundido y puntudo & - & - & - & - & 1 & AjMa03 \\
\hline \multicolumn{7}{|c|}{ Forma del fruto con la unión con el pedicelo } \\
\hline Obtuso & 8 & 5 & 1 & 2 & - & \\
\hline Truncado & 32 & 32 & 19 & 24 & - & \\
\hline Cordado & - & - & - & - & 1 & AjMa03 \\
\hline Lobulado & - & - & - & - & 1 & AjCo62 \\
\hline
\end{tabular}

EU: estados únicos; GI, GII, GIII, GIV, GV: grupos obtenidos a partir del método de Ward (1963) e índice de Gower (1971); *valores resaltados corresponden a estados únicos encontrados en el germoplasma de estudio. 
Continuacion, Tabla 4. Descriptores cualitativos de la colección de germoplasma de Capsicum de Agrosavia Cl Caribia.

\begin{tabular}{|c|c|c|c|c|c|c|}
\hline \multirow[b]{2}{*}{ Categoria } & \multicolumn{6}{|c|}{ Grupos } \\
\hline & $\begin{array}{c}\mathrm{GI} \\
(40)\end{array}$ & $\begin{array}{l}\text { GII } \\
(37)\end{array}$ & $\begin{array}{l}\text { GIII } \\
(20)\end{array}$ & $\begin{array}{l}\text { GIV } \\
\text { (26) }\end{array}$ & $\begin{array}{l}\text { GV } \\
(2)\end{array}$ & $\begin{array}{c}\text { EU } \\
\text { (accesión) }\end{array}$ \\
\hline \multicolumn{7}{|l|}{ Tipo de epidermis del fruto } \\
\hline Lisa & 39 & 37 & 20 & 26 & 2 & \\
\hline Semirrugosa & 1 & - & - & - & - & AjCo02 \\
\hline \multicolumn{7}{|l|}{ Color del fruto en estado maduro } \\
\hline Café rojizo & - & - & 1 & - & - & AjCo09 \\
\hline Rojo & 40 & 32 & 18 & 26 & 2 & \\
\hline Rojo oscuro & - & 2 & - & - & - & \\
\hline Rojo intermedio & - & 3 & 1 & - & - & \\
\hline \multicolumn{7}{|c|}{ Arrugamiento transversal del fruto } \\
\hline Levemente corrugada & 17 & 17 & 9 & 7 & 2 & \\
\hline Intermedio & 22 & 20 & 11 & 17 & - & \\
\hline Muy corrugado & 1 & - & - & 2 & - & \\
\hline
\end{tabular}

EU: estados únicos; GI, GII, GIII, GIV, GV: grupos obtenidos a partir del método de Ward (1963) e índice de Gower (1971); *valores resaltados corresponden a estados únicos encontrados en el germoplasma de estudio.

Las diferencias del GV con respecto al resto de grupos se debe a que las accesiones de este grupo corresponden a cultivares de ají dulce tipo "chino" (Capsicum annuum) que difieren a nivel de especie del resto de accesiones de la colección que pertenecen a cultivares de ají dulce tipo "topito" (Capsicum chinense).

Así mismo, al considerar la moda de los 19 descriptores cualitativos polimórficos de los grupos determinados, se encuentra que el GV difiere en $11(57,9 \%), 12(63,2 \%), 13(68,4 \%)$ y $14(73,7 \%)$ descriptores con los grupos GIII, GIV, GIl y GI respectivamente (Tabla 4).

$\mathrm{Al}$ respecto, C. annuum, $C$. chinense y $\mathrm{C}$. frutescens están integradas por características morfológicas, derivadas de parientes silvestres de diferentes especies; son potencialmente fáciles de cruzar y, por tanto, tienen la capacidad de producir híbridos interespecíficos. Sin embargo, es posible su discriminación mediante el empleo distancias combinadas (cualitativas y cuantitativas) con el método UPGMA (Gomes et al., 2019). Estos resultados concuerdan con los obtenidos por Sudré et al. (2010), donde la caracterización morfo-agronómica usando simultáneamente variables morfológicas (cualitativas) y agronómicas (cuantitativas) permitió la separación de especies del género Capsicum en la colección de estudio.

Las accesiones con estados únicos adquieren relevancia en la conservación, ya que estos pueden corresponder a atributos con valor potencial (Medina et al., 2006). Un ejemplo de ello es el carácter color del fruto, el cual, es un atributo importante tanto para el mercado de consumo fresco como para la agroindustria de procesados (Pérez-Gálvez et al., 2004).

En este sentido autores como Duran (2013), Lang et al. (2004) y Junior e Silva et al. (2013) indican que se ha incrementado la demanda de frutos de colores variados en Capsicum; por ende, es considerado como atributo de importancia económica en el 
desarrollo de cultivares mejorados. Adicionalmente Osawaru et al. (2015), expresan que el polimorfismo único puede ser útil como marcador de otros atributos estrechamente ligados, es decir, que bajo esta condición algunos atributos morfológicos pueden ser considerados como marcadores genéticos.

La tabla 5, relaciona los descriptores cuantitativos considerados en el estudio. El mayor rendimiento por planta se obtuvo en las accesiones que componen el GIV con 861,37 g/planta; seguido del GI (719,1 g/planta), GV (699,79 g/planta), GIII (666,31 g/planta) y GII (451,02 g/planta). El GV fue superior en características como precocidad (DFlor=
92,5 días; Dfruc $=110$ días), dimensiones de fruto $(\mathrm{LonF}=6,36 \mathrm{~cm} ; \mathrm{AnF}=2,68 \mathrm{~cm}$; $\mathrm{EPF}=1,95 \mathrm{~mm}$ ) y peso promedio de fruto $(\mathrm{PPF}=8,49 \mathrm{~g})$; pero inferior al resto de grupos en caracteres como: NFP (86,08 frutos/planta), DTall $(15,24 \mathrm{~mm})$, dimensiones planta $(\mathrm{AnP}=67,1 \mathrm{~cm} ; \mathrm{AltP}=48,8 \mathrm{~cm} ; \mathrm{NRam}=3,2$ ramas) y dimensiones de hoja $(\mathrm{AnH}=2,51$ $\mathrm{cm}$; $\mathrm{LonH}=6,23 \mathrm{~cm}$ ). La superioridad en rendimiento de las accesiones que componen el GIV, puede explicarse a características como precocidad (Dfruc $=135,6$ días) y por consiguiente mayor número de cosechas $(7,08$ cosechas); componentes del rendimiento como $\operatorname{PPF}(5,2$ g) y NFP (172,75 frutos/planta); y dimensiones del fruto $(\mathrm{LonF}=5,4 \mathrm{~cm} ; \mathrm{AnF}=2,4$ $\mathrm{cm})$.

Tabla 5. Descriptores cuantitativos determinados en la colección de germoplasma de Capsicum spp de Agrosavia, Cl Caribia.

\begin{tabular}{|c|c|c|c|c|c|}
\hline \multirow{2}{*}{ DESCRIPTOR } & \multicolumn{5}{|c|}{ Grupos } \\
\hline & GI & GII & GIII & GIV & GV \\
\hline Número de Cosechas NCos & $6,5 \pm 1,5$ & $5,0 \pm 1,1$ & $6,8 \pm 1,0$ & $7,1 \pm 1,2$ & $7,3 \pm 2,0$ \\
\hline Rendimiento Rend & $719,1 \pm 218,5$ & $451,0 \pm 163,8$ & $666,3 \pm 236,1$ & $861,4 \pm 265,1$ & $699,8 \pm 34,7$ \\
\hline Peso promedio de fruto PPF & $5,0 \pm 0,7$ & $4,7 \pm 0,5$ & $4,3 \pm 0,7$ & $5,2 \pm 0,9$ & $8,5 \pm 0,1$ \\
\hline Número de frutos por planta NFP & $150,4 \pm 50,6$ & $100,4 \pm 33,9$ & $157,2 \pm 45,7$ & $172,8 \pm 66,1$ & $86,1 \pm 4,4$ \\
\hline Ancho de la hoja AnH & $6,3 \pm 0,6$ & $5,2 \pm 0,7$ & $5,0 \pm 0,8$ & $5,1 \pm 0,7$ & $2,5 \pm 0,6$ \\
\hline Longitud de la hoja LonH & $12,2 \pm 1,0$ & $10,1 \pm 1,3$ & $10,1 \pm 1,4$ & $10,5 \pm 1,4$ & $6,2 \pm 0,3$ \\
\hline Número de ramas NRam & $5,8 \pm 0,8$ & $5,3 \pm 0,6$ & $5,5 \pm 0,6$ & $5,4 \pm 0,8$ & $3,2 \pm 0,8$ \\
\hline Ancho de planta AnP & $73,3 \pm 11.1$ & $70,7 \pm 10,8$ & $82,0 \pm 9,8$ & $92,7 \pm 11,1$ & $67,1 \pm 10,3$ \\
\hline Altura de planta AltP & $87,0 \pm 8,0$ & $76,2 \pm 11,8$ & $82,5 \pm 9,4$ & $95,4 \pm 17,6$ & $48,8 \pm 17,5$ \\
\hline Diámetro del tallo DTall & $20,6 \pm 2,7$ & $19,6 \pm 4,1$ & $18,9 \pm 2,3$ & $25,7 \pm 4,3$ & $15,2 \pm 0,7$ \\
\hline Longitud del tallo LonT & $7,4 \pm 2,9$ & $7,8 \pm 2,8$ & $13,3 \pm 3,0$ & $12,2 \pm 3,6$ & $10,7 \pm 2,0$ \\
\hline Número de lóculos NLoc & $2,1 \pm 9,1$ & $2,0 \pm 0,1$ & $2,0 \pm 0,1$ & $2,0 \pm 0,1$ & $2,8 \pm 0,3$ \\
\hline Ancho de fruta AnF & $2,4 \pm 0,2$ & $2,4 \pm 0,2$ & $2,5 \pm 0,6$ & $2,4 \pm 0,2$ & $2,7 \pm 0,1$ \\
\hline Longitud del fruto LonF & $5,4 \pm 0,4$ & $5,1 \pm 0,5$ & $4,9 \pm 0,8$ & $5,4 \pm 0,4$ & $6,4 \pm 1,9$ \\
\hline Espesor de la pared del fruto EPF & $1,5 \pm 0,2$ & $1,5 \pm 0,1$ & $1,5 \pm 0,2$ & $1,6 \pm 0,2$ & $1,9 \pm 0,0$ \\
\hline Días a floración DFlor & $133,8 \pm 9,6$ & $139,2 \pm 8,2$ & $127,2 \pm 10,5$ & $121,2 \pm 8,7$ & $92,5 \pm 3,5$ \\
\hline Días de fructuficación DFruc & $148,3 \pm 14,2$ & $154,7 \pm 11,8$ & $139,7 \pm 13,0$ & $135,6 \pm 8,5$ & $110,0 \pm 9,9$ \\
\hline
\end{tabular}

NCos: número de cosechas(\#); Rend: rendimiento (g/planta); PPF: peso promedio del fruto (g); NFP: número de frutos por planta(\#); AnH: ancho de la hoja (cm); LonH: longitud de la hoja (cm); NRam: número de ramas (\#); AnP: ancho de planta $(\mathrm{cm})$; AltP: altura de planta $(\mathrm{cm})$; DTall: diámetro del tallo $(\mathrm{mm})$; LonT: longitud del tallo (cm); NLoc: número de lóculos (\#); AnF: ancho de fruto (cm); LonF: longitud del fruto (cm); EPF: espesor de la pared del fruto. (mm); DFlor: días a floración (día); DFruc: días a fructificación (día). 


\section{CONCLUSIONES}

El análisis mixto de los datos de caracterización morfo-agronómica permitieron formar cinco grupos de accesiones de ají dulce y separar las especies del género Capsicum en la colección de estudio.

Los procesos continuos de selección por parte de los agricultores han influido en la fijación de caracteres morfológicos en los cultivares regionales de ají dulce.

La variabilidad fenotípica determinada indica un potencial para su uso en programas de mejoramiento genético, especialmente para caracteres de interés como calidad de fruto (forma, longitud y peso medio del fruto), producción (rendimiento y número de frutos por planta) y arquitectura de planta (habito de crecimiento).

\section{AGRADECIMIENTOS}

Corporación Colombiana de Investigación Agropecuaria - Agrosavia y al Ministerio de Agricultura y Desarrollo Rural por la financiación con fondos públicos para esta investigación.

\section{Conflicto de Intereses}

Los autores declaran que es un trabajo orginal y no existío conflicto de intereses de ningún tipo en la elaboración y publicación del manuscrito.

\section{REFERENCIAS}

Agronet. 2019. Área Cosechada, Producción y Rendimiento de Ají dulce 2006-2018. En: Agronet

https://www.agronet.gov.co/estadistica/ Paginas/home.aspx

Araújo, L., Neves, L., Sousa, D., Zeviani, W., Silva, L. and Marostega, T. 2019. Biochemical descriptors: importance of the genetic divergence study in peppers. Horticultura Brasileira 37: 210-214. doi.org/10.1590/s0102-053620190212
Barchenger, D. and Bosland, P. 2019. Wild Chile Pepper (Capsicum L.) of North America. In: Greene S., Williams K., Khoury C., Kantar M., Marek L. (eds) North American Crop Wild Relatives. Springer, Cham, (2): 225-242.

Barchenger, D., Naresh, P. and Kumar, S. 2019. Genetic Resources of Capsicum. In: Ramchiary, N., Kole C. (eds) The Capsicum Genome. Compendium of Plant Genomes. Springer, Cham, p. 9-23.

Costa, G., Da Silva, B., Lopes, A., Carvalho, L. and Gomes, R. 2019. Selection of pepper accessions with ornamental potential. Rev. Caatinga, 32(2): 566 - 574.

Di Rienzo, J., Casanoves, F., Balzarini, M., Gonzalez, L., Tablada, M. and Robledo, C. 2017. InfoStat software estadistico versión estudiantil. Grupo InfoStat, FCA, Universidad Nacional de Córdoba, Argentina.

Duran, F. 2013. Cultivo de pimientos, chiles y ajíes. Primera edición, Grupo Latino, Bogotá, 339p.

Esbaugh, W. 1993. Peppers: history and exploitation of a Serendipitous new crop Discovery. pp. 132-139. In: Janick, J. \& Simon, J. (Eds.). New Crops. 1th Ed. Wiley, New York.

FAO. 2010. The Second Report on the State of the World's: Plant Genetic Resources for Food and Agriculture. Rome, Italy. 399p.

FAOSTAT. 2019. Datos sobre alimentación y agricultura: área cosecha, producción y rendimientos de chiles, pimientos picantes, pimientos (verdes) a nivel mundial 2017. En FAOSTA

http://www.fao.org/faostat/es/\#home

Franco, T. e Hidalgo, R. 2003. Análisis estadístico de datos de caracterización morfológica de recursos fitogenéticos. $1 \mathrm{a}$ ed. IPGRI. Cali, pp. 40-84. 
Guzmán, F., Moore, S., De Vicente, C. and Jahn, M. 2019. Microsatellites to enhance characterization, conservation and breeding value of Capsicum germplasm. Genet Resour Crop Evol. doi.org/10.1007/s10722-019-00801-w

Gomes, G., Baba, V., Santos, O., Sudré, C., Bento, C., Rodrigues, R. and Gonçalves, L. 2019. Combinations of distance measures and clustering algorithms in pepper germplasm characterization. Horticultura Brasileira 37: 172-179

Hidalgo, R. y Vallejo, F. 2014. Bases para el estudio de los recursos genéticos de especies cultivadas. 1a Ed. Universidad Nacional de Colombia sede Palmira. Cali. 286p.

IPGRI, AVRDC. y CATIE. 1995. Descriptores para Capsicum spp. 1a ed. IPGRI, AVRDC y CATIE. Roma, Italia. 47 p.

Jesus, R., Santos, G., Piccin, A., Balsalobre, T., Sala, F. and Carneiro, M. 2019. Characterization of pepper accessions using molecular markers linked to pungency and SSR. Horticultura Brasileira 37: 152-160.

Junior e Silva, W., Carvalho, S. and Duarte, J. 2013. Identification of minimum descriptors for characterization of Capsicum spp. germplasm. Horticultura Brasileira 31: 190-202.

Lang, Y., Yanagawa, S., Sasanuma, T. and Sasakuma, T. 2004. Orange fruit color in Capsicum due to deletion of Capsanthin - capsorubin synthesis gene. Breeding Science 54: 33-39.

Marame, F., Dessalegne, C., Fininsa, C. and Sigvald, R. 2009. Heterosis and heritability in crosses among Asian and Ethiopian parents of hot pepper genotypes. Euphytica 168: 235-247.
Medina, C., Lobo, M. y Farley, A. 2006. Variabilidad fenotípica en poblaciones de ají y pimentón de la colección colombiana del género Capsicum. Revista Corpoica Ciencia y Tecnología Agropecuaria 7(2): 25-39.

Mendes, M., Santos, M., Santos, M., Cameron, L., Ferreira, M. and Gonçalves, E. 2019. Characterization of pepper (Capsicum baccatum) - A potential functional ingredient. LWT-Food Science and Technology, 112: 1-9.

MINCIT. 2013. Plan de negocios de ají: Programa de transformación productiva. En: MINCIT.

https://www.colombiaproductiva.com/ ptp-capacita/publicaciones/sectoriales/ publicaciones-frutas-y-sus-derivados/ plan-de-negocios-de-aji-2013

Nicolaï, M., Cantet, M., Lefebvre, V., SagePalloix, A.M. and Palloix, A. 2013. Genotyping a large collection of pepper (Capsicum spp.) with SSR loci brings new evidence for the wild origin of cultivated C. annuum and the structuring of genetic diversity by human selection of cultivar types. Genet. Resour. Crop Evol., 60: 2375-2390.

Occhiuto, P., Peralta, I., Asprelli, P. y Galmarini, C. 2014. Caracterización del germoplasma de Capsicum recolectado en el noreste de Argentina basado en rasgos morfológicos y de calidad. Agriscientia 31(2): 63-73.

Osawaru, M., Ogwu, M. and Aiwansoba, R. 2015. Hierarchical Approaches to the Analysis of Genetic Diversity in Plants: A Systematic Overview. In: University of Mauritius Research Journal.

https://www.ajol.info/index.php/umrj/ article/view/122070/111548

Paran, I. and Van Der Knaap, E. 2007. Genetic and molecular regulation of fruit and plant domestication traits in tomato and pepper. J. Exp. Bot., 58: 3841-3852. 
Pérez-Gálvez, A., Hornero-Méndez, D. and Mínguez-Mosquera, M. 2004. Changes in the carotenoid metabolism in Capsicum fruits during application of modelized slow dry in process for paprika production. Journal of Agricultural and Food Chemistry 52(3): 518-522.

Pickersgill, B. 1997. Genetic resources and breeding of Capsicum spp. Euphytica 96: 129-133. doi.org/10.1023/A:1002913228101

Pinto, M., Correa, E., Páez, A., Guzmán, N. y Baquero, C. 2013. Modelo productivo de ají topito (Capsicum spp.) para la región Caribe. 1a ed. Corpoica, Bogotá. 272 p.

Rahevar, P., Patel, J., Kumar, S. and Acharya, R. 2019. Morphological, biochemical and molecular characterization for genetic variability analysis of Capsicum annuum. Vegetos, 32:131-141.

Srivastava, A. and Mangal, M. 2019. Capsicum Breeding: History and Development. In: Ramchiary, N., Kole C. (eds) The Capsicum Genome. Compendium of Plant Genomes. Springer, Cham, p. 2555.

Sudré, C., Gonçalves, L., Rodrigues, R., Amaral, A., Riva-Souza, E. and Bento, S. 2010. Genetic variability in domesticated Capsicum spp. as assessed by morphological and agronomic data in mixed statistical analysis. Genetics and Molecular Research 9(1): 283-294.
Tang, H., Sezen, U. and Paterson, A. 2010. Domestication and plant genomes. Curr. Opin. Plant Biol., 13: 160-166.

Vallejo, F. y Estrada, E. 2013. Mejoramiento genético de plantas. 2a Ed. Universidad Nacional de Colombia sede Palmira. Cali. 454p.

Villota-Cerón, D., Bonilla, M., Carmen, H., Jaramillo, J. y García, M. 2012. Caracterización morfológica de introducciones de Capsicum spp. existentes en el Banco de Germoplasma activo de Corpoica C.I. Palmira, Colombia. Acta Agronómica 61(1): 1626.

Votava, E., Nabhan, G. and Bosland, P. 2002. Genetic diversity and similarity revealed via molecular analysis among and within an in situ population and ex situ accessions of chiltepín (Capsicum annuum var. glabriusculum). Conservation Genetics 3: 123-129.

Votava, E., Baral, J. and Bosland, P. 2005. Genetic diversity of chile (Capsicum annuum var. annuum L) landraces from Northern New Mexico, Colorado and Mexico. Economic Botany 59(1): 8-17. 